\title{
Numerical Study of Natural Convective Heat and Mass Transfer in an Inclined Porous Media
}

\author{
A. Latreche \\ Laboratory of Energetic Physics \\ Faculty of Exact Sciences \\ University Frères Mentouri Constantine 1 \\ Constantine, Algeria \\ abdelkrim.latreche@gmail.com
}

\author{
M. Djezzar \\ Laboratory of Energetic Physics \\ Faculty of Exact Sciences \\ University Frères Mentouri Constantine 1 \\ Constantine, Algeria \\ mdjezzar@umc.edu.dz
}

\begin{abstract}
In this study, two dimensional natural convection heat and mass transfer generated in an inclined rectangular porous cavity filled with Newtonian fluid has been investigated numerically. The cavity is heated and cooled along horizontal walls while the solutal gradient is imposed horizontally. The physical model for the momentum conservation equation makes use of the Darcy model, and the set of coupled equations is solved using a finite volume approach. The successive-under-relaxation (SUR) method is used in the solution of the stream function equation. The results are presented graphically in terms of streamlines, isotherms and iso-concentrations. The heat and mass transfer rate in the cavity is measured in terms of the average Nusselt and Sherwood numbers for various non-dimensional parameters.
\end{abstract}

Keywords-natural convection; heat and mass transfer; finite volumes; inclined cavity; porous media

\section{INTRODUCTION}

Double-diffusive natural convection in a fluid-saturated porous medium is of significant interest due to its various applications in different fields such as astrophysics, oceanography, chemical processes etc.. It is also met in many engineering applications such as the migration of moisture contained in fibrous insulation, drying processes, chemical transport in packed-bed reactors, grain storage installations, food processing, underground disposal of nuclear waste etc.. A summary of the work done in the past is presented in [1-8]. Including different kinds of boundary conditions and methods of solutions, most studies are concerned with two kinds of heat and mass gradients: the first kind is gradients imposed horizontally along the enclosure [9-14] and the second is gradients imposed vertically [15-18]. Relatively less attention has been given to the phenomenon of double-diffusive natural convection where cross gradients of temperature and concentration are imposed [19-21] and, to the best of our knowledge, no work is cited in an inclined saturated porous medium where this kind of gradient is imposed, which is the subject of this work.

\section{MATHEMATICAL MODELING}

Consider steady two-dimensional laminar natural convection in an inclined rectangular porous cavity of length $L$ and height $H$. The horizontal walls of the cavity are impermeable to the transport of solute and they are subjected to temperatures $\mathrm{T}_{h}$ and $\mathrm{T}_{l}$ at bottom and top walls, respectively $\left(\mathrm{T}_{h}>\mathrm{T}_{l}\right)$. The vertical walls are thermally insulated and they are subjected to fixed concentrations, high concentration at the left $\mathrm{S}_{h}$ and low concentration $\mathrm{S}_{l}$ at the right wall of the cavity as depicted in Figure 1. Hypotheses of incompressible and laminar flow are considered, and the saturated porous medium is assumed isotropic and homogeneous with constant thermo physical properties. Interaction between the thermal and concentration gradients, (Soret and Dufour effects) are neglected. The flow is driven by combined buoyancy forces because of temperature and concentration variations. So, the density is taken as a function of both temperature and concentration levels through the Boussinesq approximations:

$$
\rho=\rho_{0}\left[1-\beta_{T}\left(T-T_{l}\right)-\beta_{S}\left(S-S_{l}\right)\right]
$$

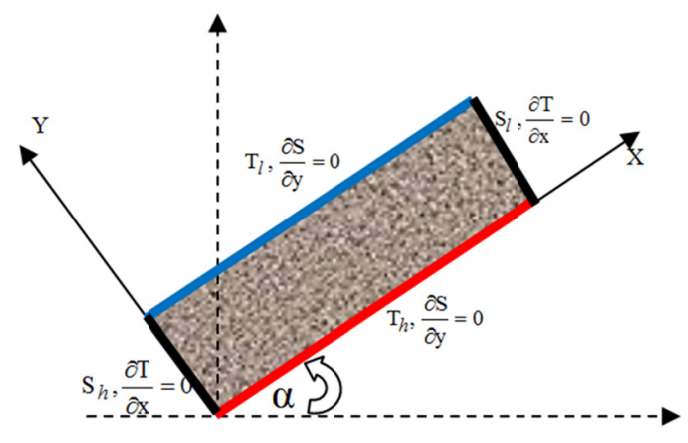

Fig. 1. Physical model and geometry

Under the above assumptions, the dimensionless equations for conservation of momentum, energy and species transport in rectangular coordinate systems are given by (2)-(4):

$$
\begin{aligned}
\frac{\partial^{2} \psi}{\partial x^{2}}+\frac{\partial^{2} \psi}{\partial y^{2}} & =-R a *\left(\cos \alpha\left(\frac{\partial T}{\partial x}+N \frac{\partial S}{\partial x}\right)\right. \\
& \left.-\sin \alpha\left(\frac{\partial T}{\partial y}+N \frac{\partial S}{\partial x}\right)\right)
\end{aligned}
$$




$$
\begin{aligned}
& \frac{\partial \psi}{\partial y} \cdot \frac{\partial T}{\partial x}-\frac{\partial \psi}{\partial x} \cdot \frac{\partial T}{\partial y}=\left(\frac{\partial^{2} T}{\partial x^{2}}+\frac{\partial^{2} T}{\partial y^{2}}\right) \\
& \frac{\partial \psi}{\partial y} \cdot \frac{\partial S}{\partial x}-\frac{\partial \psi}{\partial x} \cdot \frac{\partial S}{\partial y}=\frac{1}{L e}\left(\frac{\partial^{2} S}{\partial x^{2}}+\frac{\partial^{2} S}{\partial y^{2}}\right)
\end{aligned}
$$

where the dimensionless variables are:

$$
\begin{gathered}
x=\frac{\mathrm{x}}{H}, \quad y=\frac{\mathrm{y}}{H}, \quad \psi=\frac{\psi}{a}, \quad T=\frac{\mathrm{T}-\mathrm{T}_{l}}{\mathrm{~T}_{h}-\mathrm{T}_{l}} \\
S=\frac{\mathrm{S}-\mathrm{S}_{l}}{\mathrm{~S}_{h}-\mathrm{S}_{l}}
\end{gathered}
$$

The dimensionless boundary conditions are:

$$
\begin{aligned}
& \forall x \text { and } y=0: T=1, \frac{\partial S}{\partial y}=0 \text { and } \psi=0 \\
& \forall x \text { and } y=1: T=0, \frac{\partial S}{\partial y}=0 \text { and } \psi=0 \\
& \forall y \text { and } x=0: S=1, \frac{\partial T}{\partial x}=0 \text { and } \psi=0 \\
& \forall y \text { and } x=A: S=0, \frac{\partial T}{\partial x}=0 \text { and } \psi=0
\end{aligned}
$$

The present problem is governed by 5 parameters: the buoyancy ratio $N$, the Lewis number $L e$, Darcy-modified Rayleigh number $R a^{*}$, the inclination angle $\alpha$ and the aspect ratio $A$. The average values of Nusselt number evaluated on the bottom wall and Sherwood number evaluated on the left side wall are given by:

$$
N u=-\left.\frac{1}{A} \int_{0}^{A} \frac{\partial T}{\partial y} d x\right|_{y=0}, S h=-\left.\int_{0}^{1} \frac{\partial S}{\partial x} d y\right|_{x=0}
$$

\section{NUMERICAL SOLUTION}

The volume finite method [22] is employed to solve numerically the governing equations together with the boundary conditions. The computation domain is divided into rectangular control volumes with one grid located at the center of the control volume that forms a basic cell. The set of conservation equations are integrated over the control volumes, leading to a balance equation for the fluxes at the interface. The iterative process, employed to find the stream function, temperature and concentration fields, was repeated until the following convergence criterion was satisfied:

$$
\frac{\sum_{i} \sum_{j}\left(\Phi_{i, j}^{\text {new }}-\Phi_{i, j}^{\text {old }}\right)}{\sum_{i} \sum_{j} \Phi_{i, j}^{\text {new }}} \leq 10^{-6}
$$

where $\Phi$ stands for $\Psi, T$ and $S$. The subscripts $\mathrm{i}$ and $\mathrm{j}$ denote grid locations in the $(\mathrm{x}, \mathrm{y})$ plane. A further decrease of the convergence criteria, $10^{-6}$, does not cause any significant change in the final results. Numerical tests, using various mesh sizes, were done for the same conditions in order to determine the best compromise between accuracy of the results and computer time. A mesh size of $121 \times 61$ was adopted. The accuracy of the code was checked, modifying the thermal and solutal boundary conditions, to reproduce the results reported in [20]. Good agreement can be seen from Table I with a maximum deviation of about $3.4 \%$.

TABLE I. VALIDATION OF THE NUMERICAL CODE

\begin{tabular}{|c|c|c|c|c|c|c|}
\hline \multirow{2}{*}{ Le } & \multicolumn{2}{|c|}{$\boldsymbol{\psi}_{\max }$} & \multicolumn{2}{c|}{ Nu } & \multicolumn{2}{c|}{ Sh } \\
\cline { 2 - 7 } & $\begin{array}{c}\text { Present } \\
\text { work }\end{array}$ & {$[\mathbf{2 0 ]}$} & $\begin{array}{c}\text { Present } \\
\text { work }\end{array}$ & {$[20]$} & $\begin{array}{c}\text { Present } \\
\text { work }\end{array}$ & {$[20]$} \\
\hline 0.1 & 11.625 & 11.706 & 4.484 & 4.633 & 1.209 & 1.221 \\
\hline 1 & 9.505 & 9.609 & 4.130 & 4.276 & 4.840 & 5.086 \\
\hline 10 & 9.104 & 9.171 & 3.983 & 4.078 & 15.870 & 17.02 \\
\hline \multicolumn{6}{c|}{$\alpha=0, R a^{*}=200, \mathrm{n}=0.3$ and various $l e$, in terms of $\psi_{\mathrm{Max}}, \mathrm{n} u$ and $\mathrm{s} h$}
\end{tabular}

\section{RESUlTS AND DISCUSSION}

\section{A. Considered Situations}

In present work, the results are displayed in the form of stream, iso-thermal and iso-concentration lines to investigate the effect of inclination angle $\left(0^{\circ} \leq \alpha \leq 90^{\circ}\right)$, buoyancy ratio $(-5 \leq N \leq 5)$ and Lewis number $(0.1 \leq L e \leq 10)$ while Darcymodified Rayleigh number, aspect ratio and Prandtl number are taken as 200, 2 and 0.71 respectively. The rate of heat and mass transfer at different conditions in the cavity is measured in terms of the average Nusselt and Sherwood numbers.

\section{B. Flow Structure, Temperature and Concentration Fields, and Heat and Mass Transfer Visualization.}

To show the effects of the inclination angle $\alpha$, isotherms, stream functions and iso-concentration lines are presented in Figure 2 for $L e=10$ and $N=0.1$. For no inclination angle $\left(\alpha=0^{\circ}\right)$ four regions of important concentration gradients can be observed. Two of these regions are located in the vicinity of the upper part of the right wall and the lower part of the left wall where the cells exchange important quantity of solute with these boundaries. The two other regions are located in the vicinity of the upper and the lower central parts of the enclosure, in the interface between the cells where the solute exchange is by diffusion. For $0^{\circ}<\alpha<15^{\circ}$ the previous regions exchange just their positions (the upper becomes lower and vice versa). The vortex strength of the fluid in the porous medium is lower when $\alpha \approx 15^{\circ}$ and it increases when the angle of inclination decreases.

When $\alpha>15^{\circ}$ the secondary circulations disappear and the vortex strength of the fluid in the porous medium increases with the increase of the angle of inclination. The temperature field obtained shows the presence of thermal boundary layers near the upper and lower walls and parallel to the two other walls stratification of the temperature in the core region. The two regions of important concentration gradients located in the vicinity of the upper and the lower central parts of the enclosure disappeared. 


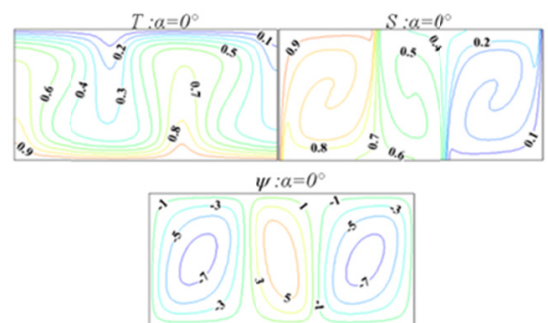

(a)

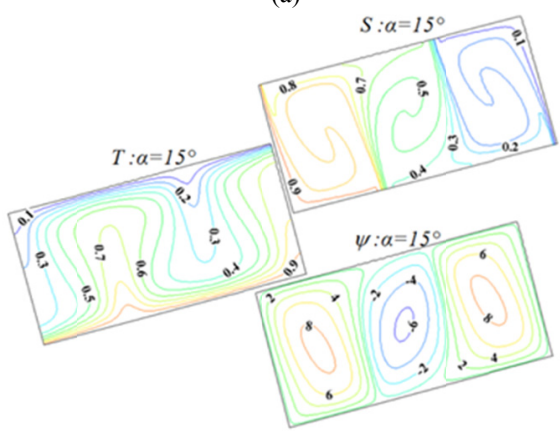

(b)

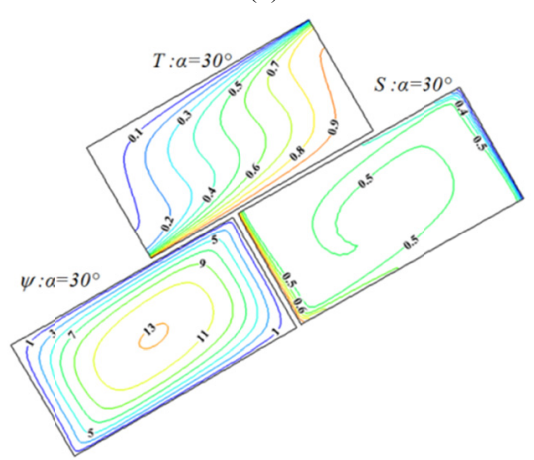

(c)

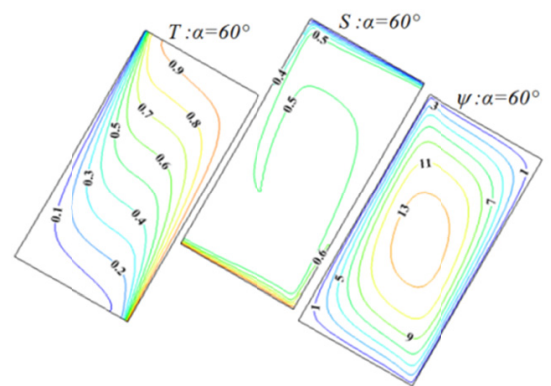

(d)

Fig. 2. Isotherms, iso-concentrationand and stream function lines for $R a^{*}=200, \operatorname{Pr}=0.71, A=2, L e=10$ and $N=0.1$ with different inclination angles.

Figure 3 presents the isotherms, iso-concentration lines and stream functions with different angles of inclination $\alpha$ for $L e=5$ and for the opposed flow $(N=-2)$. It is seen that as $\alpha$ increases the strength of the flow circulation decreases and the flow bifurcates into two weak analogical circulations. Exceeding $45^{\circ}$ engenders a decrease of the size and intensity of the lower cell in favor of the upper one. The temperature field shows that there is a tendency to the development of thermal boundary layers, this behavior decreases when the angle of inclination increases.

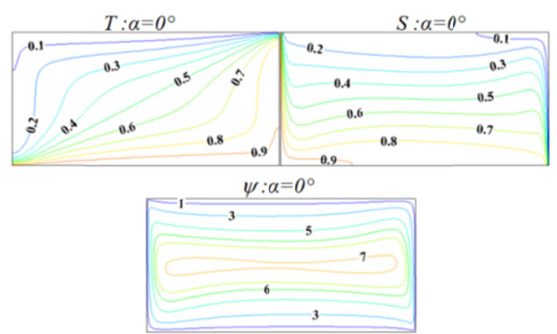

(a)

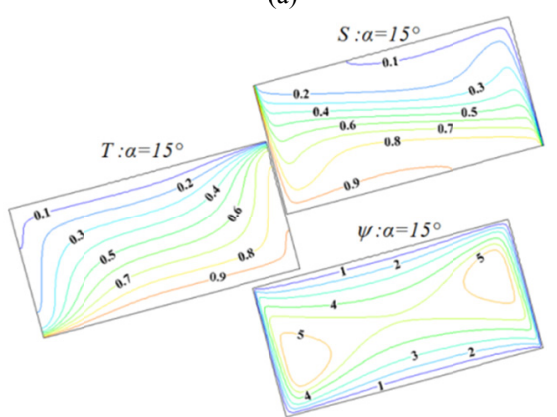

(b)

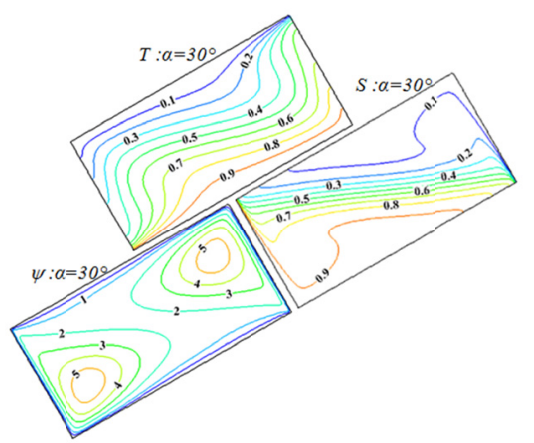

(c)

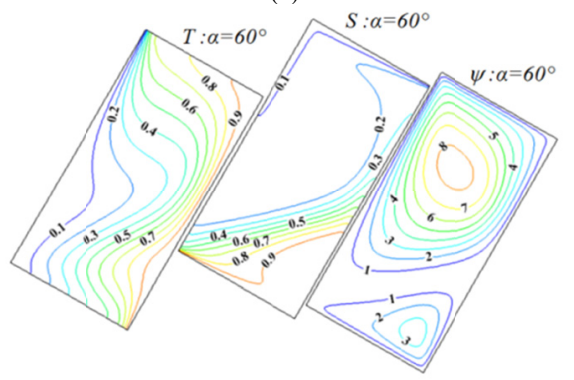

(d)
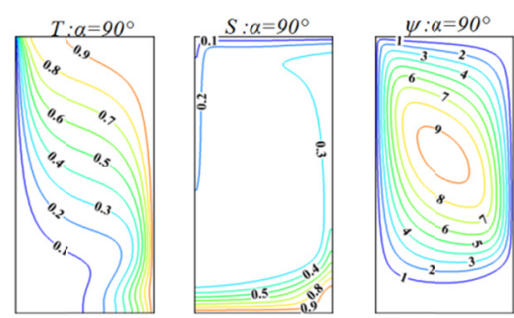

(e)

Fig. 3. Isotherms, iso-concentration and stream function lines for $R a^{*}=200, \operatorname{Pr}=0.71, A=2, L e=5$ and $N=-2$ with different inclination angles. 
The concentration field, obtained for no inclination angle $\left(\alpha=0^{\circ}\right)$, shows the presence of solutal boundary layers near the vertical walls and vertical stratification of the concentration in the core region. Three regions of important concentration gradients can be observed when the inclination angle exceeds $15^{\circ}$. Two of these regions are located in the vicinity of the upper part of the left wall and the lower part of the right wall where the cells exchange important quantity of solute with these boundaries. The third region is located in the distance which links the two previous regions at the central parts of the enclosure (the interface between the two cells) where the solute exchange is by diffusion.

\section{Heat and Mass Transfer Parameters}

To show the effects of the inclination angle, average Nusselt and Sherwood numbers are presented in Figure 4 for $R a^{*}=200, \operatorname{Pr}=0.71, A=2, N=2$ and different values of Lewis number. In general, when the Lewis number increases the Nusselt number decreases and the Sherwood number increases. Furthermore, when $\left(\alpha<30^{\circ}\right)$ the Sherwood number increases when the angle of inclination increases, while there is not a significant change on the Nusselt number. When $\left(\alpha>30^{\circ}\right)$ the Sherwood number decreases and the Nusselt number increases, when the angle of inclination increases. Previous results show that the maximum $N u$ happens when $\left(\alpha=90^{\circ}\right)$ and the maximum Sh happens when $\left(\alpha \approx 20^{\circ}\right)$.

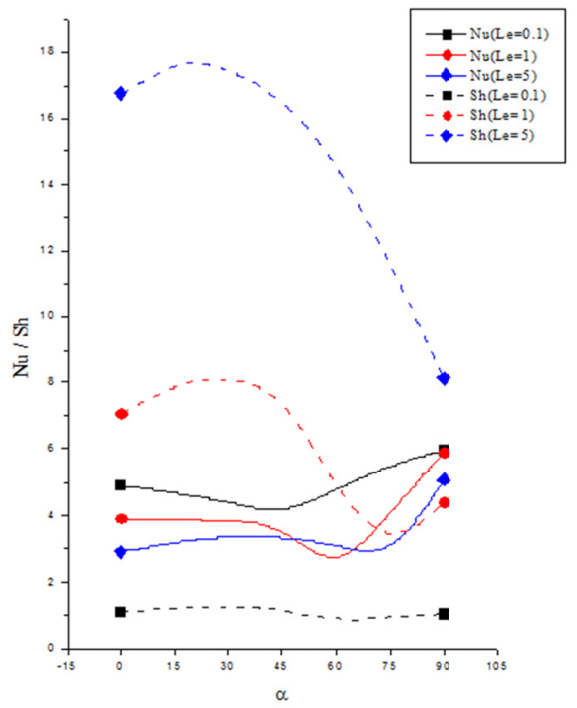

Fig. 4. Average Nusselt and Sherwood number for $R a^{*}=200, P r=0.71$, $A=2, N=2$ with different Lewis number and inclination angle values .

Figure 5 shows the effect of buoyancy ratio $N$ on average Nusselt and Sherwood Number for $R a^{*}=200, P r=0.71, A=2$, $\alpha=45^{\circ}$ for different Lewis numbers. For $(L e=0.1)$, there is not a significant change on the Nusselt and Sherwood numbers when $N$ increases until the value $N=1$ where the Nusselt and Sherwood numbers increase when $N$ increases. We can see the same behavior for $(L e=1$ and 5) until $N$ reaches the values -3 and 1, where the Nusselt and Sherwood numbers increase when $N$ increases. The Nusselt and Sherwood numbers decrease when $N$ increases only when $-1<N<1$. The maximum of both $N u$ and $S h$ happens when $N$ reaches the maximum value $(N=5)$.

\section{CONCLUSIONS}

Numerical steady of double-diffusive natural convection in an inclined porous media with vertical temperature and horizontal solutal gradients has been investigated. The generalized model has been used to solve the governing equations. A number of relevant results have been presented in this paper for different values of inclination angle of the cavity $\left(0^{\circ} \leq \alpha \leq 90^{\circ}\right)$, Lewis number $(0.1 \leq L e \leq 5)$, and the buoyancy ratio $(-5 \leq N \leq 5)$, while Darcy-modified Rayleigh number, aspect ratio and Prandtl number have been taken as 200, 2 and 0.71 respectively. Results show that the average Nusselt number decreases and the Sherwood number increases when the Lewis number increases, although, they change their behavior with the inclination angle and the buoyancy ratio. Furthermore, maximum $\mathrm{Nu}$ and $\mathrm{Sh}$ happen when $\left(\alpha=90^{\circ}\right)$ and $\left(\alpha \approx 20^{\circ}\right)$ respectively, and both of them reach maximum value when $N=5$.

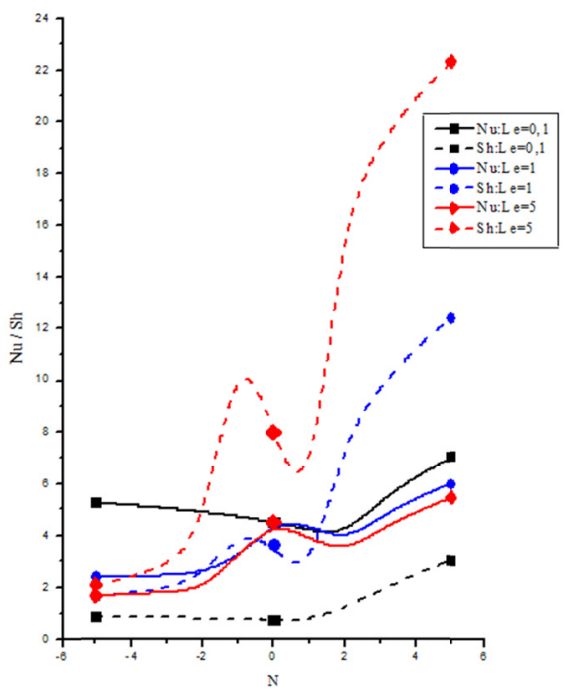

Fig. 5. Average Nusselt and Sherwood number for $R a^{*}=200, \operatorname{Pr}=0.71$, $A=2, \alpha=45^{\circ}$ with different Lewis number and buoyancy ratio values.

NOMENCLATURE

aspect ratio, $[=\mathrm{L} / \mathrm{H}]$

thermal diffusivity, $\mathrm{m}^{2} \cdot \mathrm{s}^{-1}$

solutal diffusivity, $\mathrm{m}^{2} \cdot \mathrm{s}^{-1}$

Darcy number, $\left[=\mathrm{K} / \mathrm{H}^{2}\right]$

gravitational acceleration, $\mathrm{m} \cdot \mathrm{s}^{-2}$

cavity height, $\mathrm{m}$

thermal conductivity of porous layer, $\mathrm{W} \cdot \mathrm{m}^{-1} \cdot \mathrm{K}^{-1}$

permeability of porous layer, $\mathrm{m}^{2}$

enclosure length, $\mathrm{m}$

Lewis number, $[=\mathrm{a} / \mathrm{D}]$

buoyancy ratio, $\left[=\beta_{\mathrm{S}}\left(\mathrm{S}_{\mathrm{h}}-\mathrm{S}_{\mathrm{l}}\right) / \beta_{\mathrm{T}}\left(\mathrm{T}_{\mathrm{h}}-\mathrm{T}_{1}\right)\right]$

Nusselt number

pressure, $\mathrm{kg} \cdot \mathrm{m}^{-1} \mathrm{~s}^{-2}$

dimensionless pressure, $\left[=\varepsilon^{2} \mathrm{H}^{2} \mathrm{P} / \rho_{0} \mathrm{a}^{2}\right]$

Prandtl number, $[=v / \mathrm{a}]$

Ra Rayleigh number, $\left[=\mathrm{g} \beta_{\mathrm{T}}\left(\mathrm{T}_{\mathrm{h}}-\mathrm{T}_{1}\right) \mathrm{H}^{3} / \mathrm{va}\right]$

Ra* Darcy-modified Rayleigh number, [=Ra.Da]

S dimensional concentration, $\mathrm{kg} . \mathrm{m}^{-3}$ 


$\begin{array}{ll}\mathbf{S} & \text { dimensionless concentration } \\ \mathbf{S h} & \text { average Sherwood number } \\ \mathbf{T} & \text { dimensional temperature, } \mathrm{K} \\ \mathbf{T} & \text { dimensionless temperature, }\left[=\left(\mathrm{T}-\mathrm{T}_{1}\right) /\left(\mathrm{T}_{\mathrm{h}}-\mathrm{T}_{1}\right)\right] \\ \mathbf{x , y} & \text { coordinates system, } \mathrm{m} \\ \mathbf{x}, \mathbf{y} & \text { dimensionless coordinates system, }[=\mathrm{x}(\mathrm{y}) / \mathrm{H}] \\ \alpha & \text { inclination angle, } \\ \beta_{\mathrm{S}} & \text { solutal expansion coefficient, } \mathrm{K}^{-1} \\ \beta_{\mathrm{T}} & \text { thermal expansion coefficient, } \mathrm{m}^{3} \cdot \mathrm{kg}^{-1} \\ \psi & \text { dimensional stream function, } \mathrm{m}^{2} \cdot \mathrm{s}^{-1} \\ \psi & \text { dimensionless stream function } \\ \rho & \text { density of fluid, } \mathrm{kg} \cdot \mathrm{m}^{-3} \\ \mathbf{h} & \text { higher } \\ \mathbf{l} & \text { lower } \\ \mathbf{m a x} & \text { maximum value }\end{array}$

\section{REFERENCES}

[1] R. W. Schmitt, "Double diffusion in oceanography", Annual Review of Fluid Mechanics, Vol. 26, pp. 255-285, 1994

[2] J. S. Turner, "Double diffusive phenomena", Annual Review of Fluid Mechanics, Vol. 6, pp. 37-56, 1974

[3] S. Ostrach, "Natural convection with combined driving forces", Physicochemical hydrodynamics, Vol. 1, No.4, pp. 233-247, 1980

[4] D. Nield, A. Bejan, Convection in Porous Media, Spinger, 2006

[5] D. B. Ingham, I. Pop, Transport phenomena in porous media, Elsevier, Oxford, 2005

[6] P. Vadasz, Emerging topics in heat and mass transfer in porous media, Spinger, 2008

[7] R. Viskanta, T. L. Bergman, F. P. Incropera, "Double diffusive natural convection", in: Natural Convection: Fundamentals and Applications, Hemisphere, 1985

[8] H. J. S. Fernando, "Buoyancy transfer across a diffusive interface", Journal of Fluid Mechanics, Vol. 209, pp. 1-34, 1989

[9] T. Makayssi, M. Lamsaadi, M. Naimi, M. Hasnaoui, A. Raji, A. Bahlaoui, "Natural double-diffusive convection in a shallow horizontal rectangular cavity uniformly heated and salted from the side and filled with non-Newtonian power-law fluids: the cooperating case", Energy Conversion and Management, Vol. 49, No. 8, pp. 2016-2025, 2008

[10] M .A. Teamah, "Numerical simulation of double diffusive natural convection in rectangular enclosure in the presences of magnetic field and heat source", International Journal of Thermal Sciences, Vol. 47, No. 3, pp. 237-248, 2008

[11] H. S. Harzallah, A. Jbara, K. Slimi, "Double-diffusive natural convection in anisotropic porous medium bounded by finite thickness walls: validity of local thermal equilibrium assumption", Transport in Porous Media, Vol. 103, No. 2, pp. 207-231, 2014

[12] Z. Alloui, P. Vasseur, "Convection of a binary fluid in a shallow porous cavity heated and salted from the sides", Computers \& Fluids, Vol. 81, pp. 85-94, 2013

[13] R. Nikbakhti, J. Khodakhah, "Numerical investigation of double diffusive buoyancy forces induced natural convection in a cavity partially heated and cooled from sidewalls", Engineering Science and Technology, an International Journal, Vol. 19, No. 1, pp. 322-337, 2016

[14] M. A. Teamah, M. K. Dawood, W. M. El-Maghlany, "Double diffusive natural convection in a square cavity with segmental heat sources", Scientific Research, Vol. 54, No.2, pp. 287-301, 2011

[15] V. Trevisan, A. Bejan, "Mass and heat transfer by high Rayleigh number convection in a porous medium heated from below", International Journal of Heat and Mass Transfer, Vol. 30, No. 11, pp. 2341-2356, 1987

[16] V. J. Bansod, R. K. Jadhav, "An integral treatment for combined heat and mass transfer by natural convection along a horizontal surface in a porous medium", International Journal of Heat and Mass Transfer, Vol 52, No. 11-12, pp. 2802-2806, 2009

[17] F. Zhao, D. Liu, G. Tang, "Natural convection in an enclosure with localized heating and salting from below", International Journal of Heat and Mass Transfer, Vol. 51, No. 11-12, pp. 2889-2904, 2008
[18] L. Abdelkrim, D. Mahfoud, "Convective heat and solute transfer in Newtonian fluid saturated inclined porous cavity", International Journal of Physical Research, Vol. 2, pp. 78-84, 2014

[19] A. A. Mohamad, R. Bennacer, "Natural convection in a confined saturated porous medium with horizontal temperature and vertical solutal gradients", International Journal of Thermal Sciences, Vol. 40, No. 1, pp. 82-93, 2001

[20] M. Bourich, A. Amahmid, M. Hasnaoui, "Double diffusive convection in a porous enclosure submitted to cross gradients of temperature and concentration", Energy Conversion and Management, Vol. 45, No. 1112 , pp. $1655-1670,2004$

[21] S. Roy, T. Basak, "Finite element analysis of natural convection flows in a square cavity with non-uniformly heated walls", International Journal of Engineering Science, Vol. 43, No. 8-9, pp. 668-680, 2005

[22] S. Patankar, Numerical Heat Transfer and Fluid flow, Hemisphere, 1980 